\title{
Clinicopathological features and prognoses in younger and older patients with gastric cancer
}

This article was published in the following Dove Press journal:

OncoTargets and Therapy

27 September 2017

Number of times this article has been viewed

\section{Shubin Song \\ Chunfeng Li \\ Sen $\mathrm{Li}$ \\ Xiliang Cong \\ Yingwei Xue}

Department of Gastrointestinal Surgery, Harbin Medical University

Cancer Hospital, Harbin, China
Correspondence: Yingwei Xue Department of Gastrointestinal Surgery, Harbin Medical University Cancer Hospital, Haping Road, Harbin, China Tel +8645 I 86298932

Fax +86 45I 86298932

Emailxyw80I@I63.com
Background and objectives: Patients of different ages with gastric cancer (GC) have different clinicopathological features and prognoses. The results for different crowds are limited and controversial. The aim of this study was to investigate the differences in clinicopathological features and prognoses between younger and older GC patients.

Methods: From January 2007 to December 2011, a consecutive total of 112 GC patients under 41 years old and $358 \mathrm{GC}$ patients over 69 years old who underwent gastrectomy for GC were recruited for this study. Then, the clinicopathological features and prognoses of these patients were analyzed comparatively.

Results: The gender, differentiation, carbohydrate antigen (CA) 19-9 and carcinoembryonic antigen (CEA) were significantly different between younger and older GC patients. There were more female and undifferentiated younger GC patients, and there were higher percentages of positive CA19-9 and CEA in older GC patients. The number of metastatic lymph nodes was an independent risk parameter for prognosis in younger patients, and the AJCC TNM (TumorNodes-Metastases classification by American Joint Committee on Cancer) stage, radicality and tumor size were independent risk parameters for prognosis in older GC patients. Younger GC patients have a much better prognoses with lower monocyte-to-lymphocyte ratio and higher prognostic nutritional index than older patients.

Conclusions: Younger GC patients have better immunity and nutritional status and better prognoses. The number of metastatic lymph nodes was the only risk parameter for prognosis in younger GC patients. We should take more effective treatments for younger GC patients with lymph nodes metastasis and pay more attention to the nutritional problems of older GC patients.

Keywords: gastric cancer, clinicopathological feature, age, prognosis

\section{Introduction}

Gastric cancer (GC) is a widespread malignant tumor around the world with a high incidence. ${ }^{1}$ Although we have made great progress in the treatment of GC, the prognosis after surgery still remains pessimistic. One important cause of the poor prognosis for GC patients is that most of them are diagnosed at an advanced stage with diffused filtration and lymph node metastasis, because there are no distinctive symptoms in early stage..$^{2,3}$

The age of GC patients is commonly 50-70 years, and the average onset age is $\sim 60$ years. ${ }^{4,5}$ However, younger ( $\leq 40$ years) and older ( $\geq 70$ years) GC patients still make up a biggish proportion of the total GC population. ${ }^{6,7}$ Age is an important factor affecting survival in patients with cancer, ${ }^{8}$ and there have been many reports suggesting that young GC patients tend to have a better prognosis than middle-aged patients $;, 5$ however, the differences in both prognosis and clinicopathological features 
between younger and older patients still remain to be further discussed.

Because of these factors, we retrospectively studied 112 younger GC patients and 358 older GC patients who accepted gastrectomy in our hospital, and we aimed to compare the differences in clinicopathological features and prognoses between younger and older GC patients. Recently, studies have shown that preoperative inflammatory responses and nutritional status both play important roles in tumorigenesis, progression and prognosis in various cancers..$^{9,10}$ The monocyte-to-lymphocyte ratio (MLR), a biomarker connected to a systemic inflammatory response, can reveal the immunity that hosts owned to show resistance to tumors. ${ }^{11}$ We, therefore, analyzed the biomarker MLR and prognostic nutritional index (PNI) to compare the immunity and nutritional status of younger and older GC patients.

\section{Patients and methods}

\section{Patients and data}

The study was carried out at the Harbin Medical University Cancer Hospital. From January 2007 to December 2011, a consecutive total of 1,990 patients with $112 \mathrm{GC}$ patients under 41 years old and 358 GC patients over 69 years old who underwent gastrectomies for GC were recruited for this study. The age composition of these patients is shown in Figure 1. The inclusion criteria were as follows: all of the patients 1) had preoperative gastroscopies and pathological diagnoses

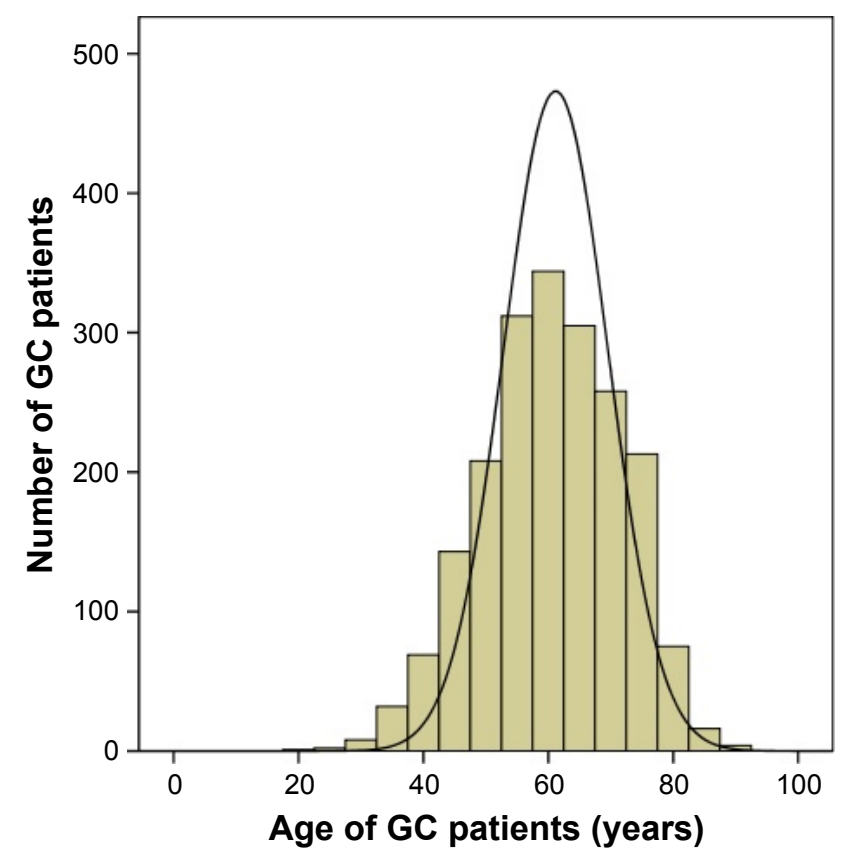

Figure I Age composition of I,990 GC patients. Abbreviation: GC, gastric cancer. of $\mathrm{GC}$; 2) never received neoadjuvant chemotherapies, radiotherapies or other anticancer treatments; 3) underwent total or subtotal gastrectomies and radical surgery (R0) patients underwent D2+ lymph nodes resection, while R1 and $\mathrm{R} 2$ patients underwent regional lymph nodes resection. The stagings were based on the 7th edition of the TNM classification (for the "Tumor-Nodes-Metastases" system) by the Union for International Cancer Control/American Joint Committee on Cancer (UICC/AJCC). ${ }^{12}$ Every patient was followed up regularly from the time of surgery until June 2016 or death (in the first two postoperative years it was every 3 months and in the following several years it was at 6-month intervals). This research had the support and approval of the Medical Ethics Committee of the Harbin Medical University Cancer Hospital, and all patients signed the informed consent for the use of their data for future study.

We recorded the age, gender, tumor depth $(\mathrm{T})$, number of metastatic lymph nodes $(\mathrm{N})$, distant metastasis (M), AJCC TNM stage, radicality, tumor size, tumor location, metastasis lymph node rate (MLNR), differentiation, ascites, carbohydrate antigen (CA) 19-9 (U/mL), carcinoembryonic antigen (CEA) (ng/mL), hemoglobin $(\mathrm{Hb})(\mathrm{g} / \mathrm{L})$, albumin count $(\mathrm{g} / \mathrm{L})$, lymphocyte count $\left(10^{9} / \mathrm{L}\right)$ and monocyte count $\left(10^{9} / \mathrm{L}\right)$. The average MLNR was 31.5\%. CA19-9 exceeding $37 \mathrm{U} / \mathrm{mL}$, CEA exceeding $5 \mathrm{ng} / \mathrm{mL}$ and $\mathrm{Hb}<131 \mathrm{~g} / \mathrm{L}$ were identified as positive. MLR was calculated as the monocyte count/lymphocyte count. ${ }^{9}$ PNI was calculated as the albumin count + lymphocyte count $\times 5 .^{10}$

\section{Statistical analysis}

A chi-squared test was used to assess the significant differences in the clinicopathological features between younger and older GC patients. Survival curves were calculated with a KaplanMeier survival analysis, and the equivalences of survival curves were analyzed by using a log-rank test. Multivariate analyses were evaluated by the Cox proportional hazards model. A Student's $t$-test was used to compare the MLR, PNI, lymphocyte, monocyte and albumin between younger and older patients. A $P<0.05$ was considered to be statistically significant. Data analysis was performed using SPSS 17.0.

\section{Results}

The clinicopathological features of 112 younger GC patients and 358 older GC patients are shown in Table 1. There were more women in the younger GC patient group than in the older GC patient group $(47.3 \%$ vs $23.5 \%, P<0.001)$. The proportion of undifferentiated adenocarcinomas in younger GC patients was significantly higher than that in older 
Table I Clinicopathological features of younger and older GC patients

\begin{tabular}{|c|c|c|c|c|}
\hline Factors & $\begin{array}{l}\text { Younger } \\
(n=\mid I 2)\end{array}$ & $\begin{array}{l}\text { Older } \\
(n=358)\end{array}$ & $\chi^{2}$ & $P$-value \\
\hline Gender & & & 23.512 & $<0.001$ \\
\hline Male & $59(52.7)$ & $274(76.5)$ & & \\
\hline Female & $53(47.3)$ & $84(23.5)$ & & \\
\hline Tumor depth & & & 0.757 & 0.86 \\
\hline TI & $7(6.3)$ & $17(4.7)$ & & \\
\hline $\mathrm{T} 2$ & $6(5.3)$ & $25(7.0)$ & & \\
\hline $\mathrm{T} 3$ & $33(29.5)$ & $102(28.5)$ & & \\
\hline $\mathrm{T} 4$ & $66(58.9)$ & $214(59.8)$ & & \\
\hline Lymph nodes & & & 6.153 & 0.104 \\
\hline No & $20(17.9)$ & $93(26.0)$ & & \\
\hline $\mathrm{NI}$ & $27(24.1)$ & $61(17.0)$ & & \\
\hline N2 & $24(2 \mid .4)$ & $93(26.0)$ & & \\
\hline N3 & $4 \mid(36.6)$ & III (3I.0) & & \\
\hline Distant metastasis & & & 2.42 & 0.085 \\
\hline Mo & $94(83.9)$ & $320(89.4)$ & & \\
\hline MI & $18(16.1)$ & $38(10.6)$ & & \\
\hline AJCC stage & & & 3.463 & 0.326 \\
\hline I & $5(4.5)$ & $25(7.0)$ & & \\
\hline ॥ & $30(26.8)$ & $89(24.9)$ & & \\
\hline III & $59(52.7)$ & $206(57.5)$ & & \\
\hline IV & $18(16.0)$ & $38(10.6)$ & & \\
\hline Radicality & & & 0.466 & 0.495 \\
\hline Ro & 85 (75.9) & $260(72.6)$ & & \\
\hline $\mathrm{R} 1$ or $\mathrm{R} 2$ & $27(24.1)$ & $98(27.4)$ & & \\
\hline Tumor size (cm) & & & 0.687 & 0.407 \\
\hline$\leq 6$ & $70(62.5)$ & $239(66.8)$ & & \\
\hline$>6$ & $42(37.5)$ & $119(33.2)$ & & \\
\hline Tumor location & & & 6.365 & 0.095 \\
\hline Upper & $12(10.7)$ & $61(17.0)$ & & \\
\hline Middle & $21(18.8)$ & $64(17.9)$ & & \\
\hline Low & $64(57.1)$ & $208(58.1)$ & & \\
\hline Whole & $15(13.4)$ & $25(7.0)$ & & \\
\hline MLNR & & & 0.022 & 0.912 \\
\hline$\leq 31.5 \%$ & $67(59.8)$ & $217(60.6)$ & & \\
\hline$>31.5 \%$ & $45(40.2)$ & |4| (39.4) & & \\
\hline Differentiation ${ }^{\mathrm{a}}$ & & & 17.662 & $<0.00 \mathrm{I}$ \\
\hline Differentiated & $6(5.4)$ & $83(23.2)$ & & \\
\hline Undifferentiated & $106(94.6)$ & $275(76.8)$ & & \\
\hline Ascites & & & 2.338 & 0.156 \\
\hline No & $105(93.8)$ & 347 (96.9) & & \\
\hline Yes & $7(6.2)$ & II (3.1) & & \\
\hline CAI9-9 (U/mL) & & & 4.76 & 0.029 \\
\hline Negative & $96(85.7)$ & $272(76.0)$ & & \\
\hline Positive & $16(14.3)$ & $86(24.0)$ & & \\
\hline CEA (ng/mL) & & & 11.865 & 0.001 \\
\hline Negative & $10 \mid(90.2)$ & $268(74.9)$ & & \\
\hline Positive & II (9.8) & $90(25.1)$ & & \\
\hline $\mathrm{Hb}(\mathrm{g} / \mathrm{L})$ & & & 1.61 & 0.204 \\
\hline Positive & $60(53.6)$ & $216(60.3)$ & & \\
\hline Negative & $52(46.4)$ & 142 (39.7) & & \\
\hline
\end{tabular}

Note: a Grades I and 2 were differentiated, and grades 3 and 4 were undifferentiated. Abbreviations: AJCC, American Joint Committee on Cancer; CA, carbohydrate antigen; CEA, carcinoembryonic antigen; GC, gastric cancer; $\mathrm{Hb}$, hemoglobin; MLNR, metastasis lymph node rate.
GC patients $(94.6 \%$ vs $76.8 \%, P<0.001)$. The positive rates of CA19-9 and CEA in younger GC patients were significantly lower than those in older GC patients $(14.3 \%$ vs $24.0 \%, 9.8 \%$ vs $\left.25.1 \%, P_{\text {all }}<0.05\right)$. However, for other features, such as tumor depth, number of metastatic lymph nodes, distant metastasis, AJCC TNM stage, surgical radicality, tumor size, tumor location, MLNR, ascites and $\mathrm{Hb}$, there were no significant differences between younger and older GC patients.

In the Kaplan-Meier survival analyses, the overall survival times of the 112 younger GC patients were much better than those of the 358 older GC patients (Figure 2, $P<0.001)$. Younger patients had a much better prognosis than older patients in the stages II $(P=0.013)$ and III $(P<0.001)$, but this was not the case in the stages I $(P=0.207)$ and IV $(P=0.069)$.

We used univariate and multivariate analyses to assess the risk parameters for prognoses in the $470 \mathrm{GC}$ patients (Table 2). The results showed that the age, tumor depth, number of metastatic lymph nodes, distant metastasis, AJCC TNM stage, surgical radicality, tumor size, MLNR, CA19-9 and CEA were all significantly associated with the prognosis in univariate analyses. However, in the multivariate analysis, only age, number of metastatic lymph nodes, AJCC TNM stage, surgical radicality and tumor size were independent risk parameters. Then, we independently analyzed the risk parameters for the prognosis in the 112 younger patients and 358 older patients. In the 112 younger patients, tumor depth, number of metastatic lymph nodes, distant metastasis, AJCC TNM stage, radicality, tumor size and MLNR were all associated with overall survival in univariate analyses, but only the number of metastatic lymph nodes was an independent risk parameter for overall survival (Table 3 ). In the 358 older GC patients, tumor depth, number of metastatic lymph nodes, distant metastasis, AJCC TNM stage, radicality, tumor size, MLNR, differentiation, ascites, CA19-9 and $\mathrm{Hb}$ were associated with overall survival in univariate analyses, and only the AJCC TNM stage, surgical radicality and tumor size were independent risk parameters for overall survival (Table 4).

Additionally, we conducted the survival analysis of MLR and PNI in younger and older GC patients, respectively. The cutoff value of MLR was the mean value that was 0.240 in younger GC patients and 0.292 in older GC patients, and so was the PNI that was 52.883 in younger GC patients and 48.862 in older GC patients. We found that both younger GC patients with lower MLR and older GC patients with higher PNI had much better prognoses (Figure 3). Comparing the 

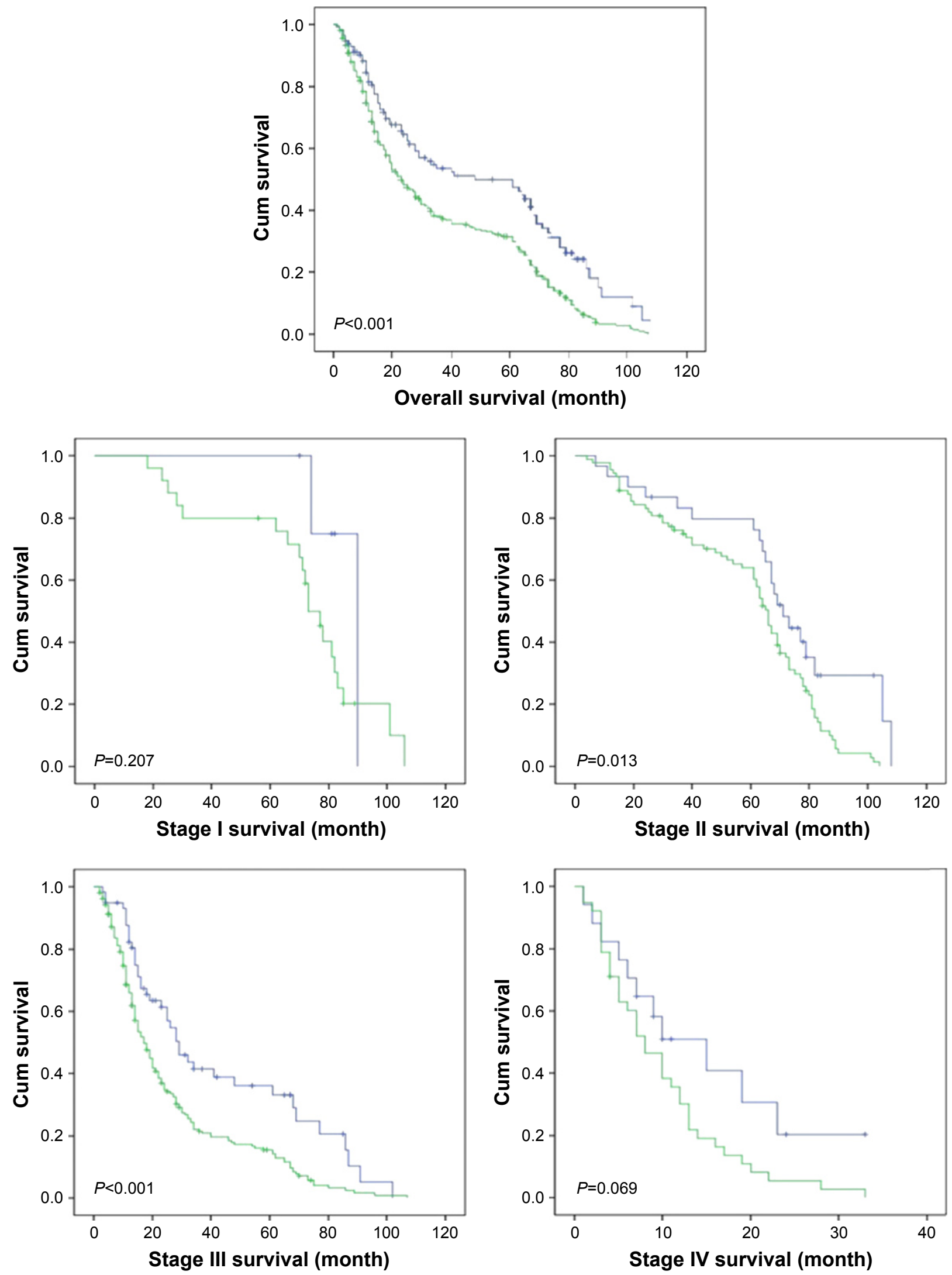

Figure 2 Comparison of overall survival between younger and older gastric cancer patients. Abbreviation: Cum, cumulative. 
Table 2 Survival analysis of the 470 younger and older GC patients

\begin{tabular}{|c|c|c|c|c|c|c|}
\hline \multirow[t]{2}{*}{ Parameter } & \multicolumn{3}{|c|}{ Univariate analysis } & \multicolumn{3}{|c|}{ Multivariate analysis } \\
\hline & $\beta$ & HR (95\% Cl) & $P$-value & $\beta$ & HR (95\% Cl) & $P$-value \\
\hline Age (younger/older) & 0.513 & $1.671(1.296-2.153)$ & $<0.001$ & 0.598 & $1.818(1.383-2.391)$ & $<0.001$ \\
\hline Gender (male/female) & -0.170 & $0.843(0.675-1.054)$ & 0.134 & -0.020 & $0.980(0.766-1.255)$ & 0.876 \\
\hline Tumor depth (TI/T2/T3/T4) & 0.372 & $1.45 \mathrm{I}(1.282-1.643)$ & $<0.001$ & -0.021 & $0.979(0.810-1.183)$ & 0.825 \\
\hline Lymph nodes (N0/NI/N2/N3) & 0.495 & $1.640(1.493-1.801)$ & $<0.001$ & 0.213 & $1.237(1.029-1.4870)$ & 0.024 \\
\hline Distant metastasis (Mo/MI) & 1.492 & $4.447(3.216-6.150)$ & $<0.001$ & 0.458 & I.58I (0.930-2.689) & 0.091 \\
\hline AJCC stage (I/II/III/IV) & 0.915 & $2.497(2.131-2.927)$ & $<0.001$ & 0.394 & $1.483(1.032-2.131)$ & 0.033 \\
\hline Radicality (R0/RI or R2) & 1.179 & $3.252(2.564-4.126)$ & $<0.001$ & 0.484 & $1.622(1.211-2.174)$ & 0.001 \\
\hline Tumor size $(\leq 6 \mathrm{~cm} />6 \mathrm{~cm})$ & 0.794 & $2.212(1.786-2.740)$ & $<0.001$ & 0.452 & $1.572(1.237-1.999)$ & $<0.001$ \\
\hline Tumor location (upper/middle/low/whole) & -0.052 & $0.950(0.836-1.078)$ & 0.424 & -0.047 & $0.954(0.843-1.08 I)$ & 0.461 \\
\hline $\operatorname{MLNR}(\leq 31.5 \% />31.5 \%)$ & 1.049 & $2.856(2.298-3.549)$ & $<0.001$ & 0.304 & $1.355(0.978-1.877)$ & 0.068 \\
\hline Differentiation (differentiated/undifferentiated) & 0.177 & $1.194(0.935-1.524)$ & 0.156 & 0.012 & $1.012(0.780-1.314)$ & 0.929 \\
\hline Ascites (no/yes) & 0.382 & $1.466(0.857-2.506)$ & 0.162 & -0.219 & $0.803(0.45 \mathrm{I}-\mathrm{I} .430)$ & 0.456 \\
\hline CAI9-9 (negative/positive) & 0.364 & $1.439(1.136-1.823)$ & 0.003 & -0.007 & $0.993(0.77|-| .279)$ & 0.958 \\
\hline CEA (negative/positive) & 0.346 & $1.423(1.114-1.792)$ & 0.004 & 0.125 & I.I $33(0.877-1.462)$ & 0.339 \\
\hline $\mathrm{Hb}(\leq 130 />130)(g / L)$ & -0.168 & $0.846(0.69 \mid-1.035)$ & 0.104 & -0.156 & $0.855(0.687-1.065)$ & 0.163 \\
\hline
\end{tabular}

Abbreviations: AJCC, American Joint Committee on Cancer; CA, carbohydrate antigen; CEA, carcinoembryonic antigen; $\mathrm{Cl}$, confidence interval; GC, gastric cancer; Hb, hemoglobin; HR, hazards ratio; MLNR, metastasis lymph node rate.

MLR and PNI values between younger and older GC patients, we found that MLR in younger patients was significantly lower $(P=0.011)$ and PNI was significantly higher $(P<0.001)$ than those in older patients (Table 5). The divergence of MLR was not significant when it was divided into stages I, II, III and IV between younger and older GC patients, while it was significant of PNI in stages II, III and IV $\left(P_{\text {all }}<0.05\right)$, but not in stage I. Tavares et al reported that lymphocyte may decline accompanied with an increase in age. ${ }^{13}$ So we calculated the differences of lymphocyte, monocyte and albumin in the two groups. We found that only albumin was significantly different between younger and older GC patients $(P<0.001)$. Finally, we compared the albumin between the two groups in different stages and found that the differences were statistically significant in stages II, III and IV $\left(P_{\text {all }}<0.05\right)$, but not in stage $\mathrm{I}(P=0.371)$.

\section{Discussion}

It is generally believed that GC is an age-related disease, and in recent decades, the number of younger and older

Table 3 Survival analysis of the II 2 younger GC patients

\begin{tabular}{|c|c|c|c|c|c|c|}
\hline \multirow[t]{2}{*}{ Parameter } & \multicolumn{3}{|c|}{ Univariate analysis } & \multicolumn{3}{|c|}{ Multivariate analysis } \\
\hline & $\beta$ & HR (95\% Cl) & $P$-value & $\beta$ & HR (95\% Cl) & $P$-value \\
\hline Gender (male/female) & -0.192 & $0.825(0.52 \mathrm{I}-1.307)$ & 0.413 & 0 & $1.000(0.573-1.746)$ & 0.999 \\
\hline Tumor depth (TI/T2/T3/T4) & 0.415 & $1.514(1.142-2.008)$ & 0.004 & 0.287 & $1.333(0.820-2.165)$ & 0.246 \\
\hline Lymph nodes (N0/NI/N2/N3) & 0.636 & $1.890(1.465-2.438)$ & $<0.001$ & 0.536 & $1.710(1.007-2.903)$ & 0.047 \\
\hline Distant metastasis (MO/MI) & 1.524 & $4.590(2.266-9.297)$ & $<0.001$ & 1.119 & $3.063(0.632-14.836)$ & 0.164 \\
\hline AJCC stage $(I / I I / I I I / I V)$ & 0.966 & 2.629 (1.799-3.842) & $<0.001$ & -0.198 & $0.82 I(0.278-2.421)$ & 0.720 \\
\hline Radicality (R0/RI or R2) & 1.502 & $4.492(2.364-8.538)$ & $<0.001$ & 0.597 & $1.816(0.683-4.830)$ & 0.232 \\
\hline Tumor size $(\leq 6 \mathrm{~cm} />6 \mathrm{~cm})$ & 0.725 & $2.065(1.266-3.367)$ & 0.004 & 0.415 & $1.515(0.838-2.740)$ & 0.169 \\
\hline Tumor location (upper/middle/low/whole) & 0.010 & $1.010(0.727-1.403)$ & 0.952 & -0.129 & $0.879(0.629-1.228)$ & 0.450 \\
\hline $\operatorname{MLNR}(\leq 31.5 \% />31.5 \%)$ & 1.163 & $3.199(1.94 I-5.274)$ & $<0.001$ & 0.154 & $1.166(0.507-2.680)$ & 0.718 \\
\hline Differentiation (differentiated/undifferentiated) & 0.244 & $1.276(0.464-3.508)$ & 0.637 & -0.052 & $0.950(0.286-3.148)$ & 0.933 \\
\hline Ascites (no/yes) & 0.146 & $1.157(0.4 \mid 5-3.23 \mathrm{I})$ & 0.780 & -1.088 & $0.337(0.07 \mathrm{I}-\mathrm{I} .608)$ & 0.173 \\
\hline CAI9-9 (negative/positive) & 0.140 & $\mathrm{I} .150(0.60 \mathrm{I}-2.200)$ & 0.673 & 0.035 & $1.036(0.496-2.162)$ & 0.926 \\
\hline CEA (negative/positive) & 0.611 & $1.843(0.938-3.622)$ & 0.076 & 0.628 & $1.873(0.680-5.164)$ & 0.225 \\
\hline $\mathrm{Hb}$ (negative/positive) & 0.444 & $1.558(0.964-2.518)$ & 0.070 & 0.364 & $\mathrm{I} .439(0.829-2.497)$ & 0.196 \\
\hline
\end{tabular}

Abbreviations: AJCC, American Joint Committee on Cancer; CA, carbohydrate antigen; CEA, carcinoembryonic antigen; Cl, confidence interval; GC, gastric cancer; Hb, hemoglobin; HR, hazards ratio; MLNR, metastasis lymph node rate. 
Table 4 Survival analysis of the 358 older GC patients

\begin{tabular}{|c|c|c|c|c|c|c|}
\hline \multirow[t]{2}{*}{ Parameter } & \multicolumn{3}{|c|}{ Univariate analysis } & \multicolumn{3}{|c|}{ Multivariate analysis } \\
\hline & $\beta$ & HR (95\% Cl) & $P$-value & $\beta$ & HR (95\% Cl) & $P$-value \\
\hline Gender (male/female) & -0.003 & $0.997(0.767-1.295)$ & 0.980 & 0.031 & $1.032(0.780-1.365)$ & 0.826 \\
\hline Tumor depth (TI/T2/T3/T4) & 0.360 & $1.433(1.247-1.648)$ & $<0.001$ & -0.110 & $0.896(0.722-1.112)$ & 0.319 \\
\hline Lymph nodes (N0/NI/N2/N3) & 0.492 & $1.635(1.479-1.807)$ & $<0.001$ & 0.169 & I.I 85 (0.965-I.453) & 0.105 \\
\hline Distant metastasis (MO/MI) & 1.563 & $4.772(3.306-6.888)$ & $<0.001$ & 0.296 & $1.345(0.745-2.426)$ & 0.325 \\
\hline AJCC stage $(I / I I / I I I / I V)$ & 0.950 & $2.586(2.167-3.087)$ & $<0.001$ & 0.528 & $1.695(1.141-2.517)$ & 0.009 \\
\hline Radicality (R0/RI or R2) & 1.097 & $2.995(2.317-3.882)$ & $<0.001$ & 0.467 & $1.595(1.164-2.186)$ & 0.004 \\
\hline Tumor size $(\leq 6 \mathrm{~cm} />6 \mathrm{~cm})$ & 0.850 & $2.339(1.84 I-2.972)$ & $<0.001$ & 0.481 & $1.618(1.233-2.122)$ & 0.001 \\
\hline Tumor location (upper/middle/low/whole) & -0.046 & $0.955(0.833-1.095)$ & 0.510 & -0.069 & $0.934(0.811-1.075)$ & 0.339 \\
\hline $\operatorname{MLNR}(\leq 31.5 \% />31.5 \%)$ & 1.045 & $2.842(2.229-3.624)$ & $<0.001$ & 0.291 & $1.337(0.930-1.923)$ & 0.116 \\
\hline Differentiation (differentiated/undifferentiated) & 0.311 & $1.364(1.055-1.765)$ & 0.018 & 0.038 & $1.038(0.791-1.364)$ & 0.786 \\
\hline Ascites (no/yes) & 0.699 & $2.012(1.069-3.787)$ & 0.030 & 0.091 & $\mathrm{I} .095(0.557-2.15 \mathrm{I})$ & 0.792 \\
\hline CAI9-9 (negative/positive) & 0.367 & $1.443(1.116-1.867)$ & 0.005 & -0.009 & $0.991(0.748-1.3130)$ & 0.949 \\
\hline CEA (negative/positive) & 0.225 & $1.253(0.969-1.619)$ & 0.085 & 0.090 & $1.094(0.834-1.436)$ & 0.517 \\
\hline Hb (negative/positive) & -0.374 & $0.688(0.547-0.865)$ & 0.001 & -0.270 & $0.764(0.594-0.982)$ & 0.035 \\
\hline
\end{tabular}

Abbreviations: AJCC, American Joint Committee on Cancer; CA, carbohydrate antigen; CEA, carcinoembryonic antigen; $\mathrm{Cl}$, confidence interval; GC, gastric cancer; $\mathrm{Hb}$, hemoglobin; HR, hazards ratio; MLNR, metastasis lymph node rate.
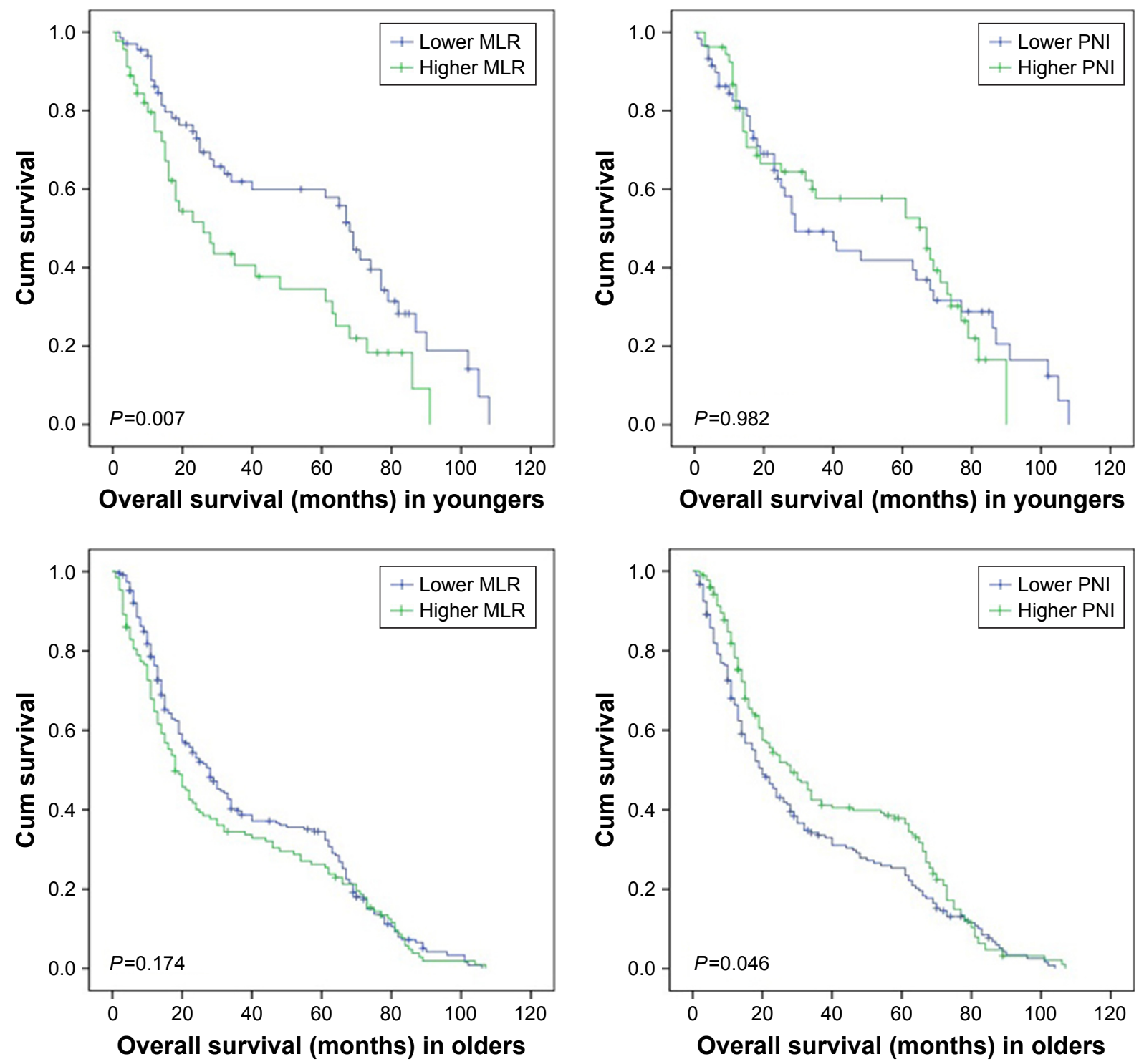

Figure 3 Survival analysis of MLR and PNI in younger and older gastric cancer patients.

Abbreviations: Cum, cumulative; MLR, monocyte-to-lymphocyte ratio; PNI, prognostic nutritional index. 
Table 5 Comparison of MLR, PNI, lymphocyte, monocyte and albumin between younger and older GC patients

\begin{tabular}{|c|c|c|c|c|c|}
\hline Stage & Biomarker & Group & $\overline{\boldsymbol{x}} \pm \mathbf{s}$ & $t$ & $P$-value \\
\hline \multirow[t]{6}{*}{ Stage I } & MLR & Younger & $0.220 \pm 0.05$ I & -0.958 & 0.346 \\
\hline & & Older & $0.30 I \pm 0.200$ & & \\
\hline & PNI & Younger & $55.250 \pm 4.226$ & 1.071 & 0.293 \\
\hline & & Older & $51.340 \pm 7.860$ & & \\
\hline & Albumin & Younger & $45.100 \pm 4.009$ & 0.909 & $0.37 I$ \\
\hline & & Older & $42.390 \pm 6.364$ & & \\
\hline \multirow[t]{6}{*}{ Stage II } & MLR & Younger & $0.210 \pm 0.093$ & -1.909 & 0.059 \\
\hline & & Older & $0.290 \pm 0.206$ & & \\
\hline & PNI & Younger & $55.890 \pm 6.413$ & 4.00 & $<0.001$ \\
\hline & & Older & $49.330 \pm 8.156$ & & \\
\hline & Albumin & Younger & $46.050 \pm 4.974$ & 4.228 & $<0.001$ \\
\hline & & Older & $40.310 \pm 6.838$ & & \\
\hline \multirow[t]{6}{*}{ Stage III } & MLR & Younger & $0.240 \pm 0.110$ & -1.453 & 0.147 \\
\hline & & Older & $0.270 \pm 0.178$ & & \\
\hline & PNI & Younger & $51.500 \pm 7.369$ & 2.201 & 0.029 \\
\hline & & Older & $49.000 \pm 7.760$ & & \\
\hline & Albumin & Younger & $42.090 \pm 6.032$ & 2.423 & 0.016 \\
\hline & & Older & $39.850 \pm 6.317$ & & \\
\hline \multirow[t]{6}{*}{ Stage IV } & MLR & Younger & $0.300 \pm 0.191$ & -1.374 & 0.175 \\
\hline & & Older & $0.400 \pm 0.269$ & & \\
\hline & PNI & Younger & $51.760 \pm 6.90 \mathrm{I}$ & 2.711 & 0.009 \\
\hline & & Older & $45.350 \pm 8.803$ & & \\
\hline & Albumin & Younger & $43.390 \pm 5.820$ & 2.678 & 0.010 \\
\hline & & Older & $38.230 \pm 7.115$ & & \\
\hline \multirow[t]{10}{*}{ All stages } & MLR & Younger & $0.240 \pm 0.123$ & -2.569 & 0.011 \\
\hline & & Older & $0.292 \pm 0.20 \mathrm{I}$ & & \\
\hline & PNI & Younger & $52.883 \pm 7.140$ & 4.730 & $<0.001$ \\
\hline & & Older & $48.862 \pm 8.060$ & & \\
\hline & Lymphocyte & Younger & $1.880 \pm 0.716$ & 1.241 & 0.215 \\
\hline & & Older & $1.780 \pm 0.75 \mid$ & & \\
\hline & Monocyte & Younger & $0.410 \pm 0.155$ & -1.649 & 0.100 \\
\hline & & Older & $0.440 \pm 0.174$ & & \\
\hline & Albumin & Younger & $43.490 \pm 5.845$ & 5.078 & $<0.001$ \\
\hline & & Older & $39.970 \pm 6.57 \mid$ & & \\
\hline
\end{tabular}

Abbreviations: GC, gastric cancer; MLR, monocyte-to-lymphocyte ratio; PNI, prognostic nutritional index.

GC patients has been increasing. It is necessary to clarify the adverse prognostic factors for patients in different age groups. In our study, we found that gender, differentiation, and CA19-9 and CEA were significantly different among younger and older GC patients. There were more women in the younger group, and the reason for this phenomenon remains unclear. There have been reports that estrogen can promote the diffusion and inhibit apoptosis in several cancers, ${ }^{14-16}$ and some have considered that this phenomenon may be related with their recent pregnancies. ${ }^{17,18}$ Jaspers et al suggested that there were more estrogen receptors in younger patients $;{ }^{19}$ however, another report demonstrated that only the number of metastatic lymph nodes was associated with estrogen receptor expression rather than other factors such as gender, age and tumor size. ${ }^{20}$ Younger GC patients generally had poor differentiation and this may be owing to that younger patients had higher infection rates of Helicobacter pylori, which lead to poor differentiation. ${ }^{21,22}$ Some reports have suggested that undifferentiated GC cells originated from gastric fundic glands, while intestinal metaplasia usually led to differentiated gastric adenocarcinoma. ${ }^{3,23}$ Higher CEA and CA19-9 levels in older patients have also been reported. ${ }^{24,25}$

In the multivariate analysis of the 470 patients, age, number of metastatic lymph nodes, AJCC TNM stage, radicality and tumor size were independent risk factors. Our study showed that younger patients had a much better prognosis than older patients, but in another study, Yukiko reported that the survival rate of young patients $(<40)$ with $\mathrm{GC}$ was the same as that of patients in their 60s. ${ }^{5}$ This result may be due to the different cohorts we analyzed. In the 112 younger GC patients, only the number of metastatic lymph nodes was an independent risk parameter according to the multivariate analysis. Yukiko also reported that younger GC patients usually have metastasis to lymph nodes. ${ }^{5}$ It is believed that undifferentiated adenocarcinomas tend to have vertical infiltration, which leads to lymph node metastasis. ${ }^{21}$

Younger GC patients have worse prognoses in general; however, in this study, we found that younger patients had a much better overall survival rate than older patients. Although we failed to show the significant prognostic differences between younger and older patients in stage I and stage IV, it may be because the patients in stage I and stage IV were too few. The difference of MLR and PNI between younger and older patients indicated that younger patients possessed better immunity and nutritional status, which may be the main reason for their longer overall survival. And the better nutritional status may play a more important role. However, more investigations are needed to explain this phenomenon. We hesitate if strengthening nutrition for older GC patients after operation could improve prognosis. This drives us to pay more attention to the nutritional problems of the elder GC patients.

There were some limitations in this study. First, this was a retrospective investigation based on a single hospital, and this may have led to some error. Second, not all of the patients who had gastrectomy in our hospital had successful consequence follow-up, which led to a smaller cohort. Third, it may be the most important that although we had told all of these patients who needed adjuvant chemotherapies to undergo subsequent treatments in our hospital or other hospitals, not all of them come back for subsequent standard treatments. So we cannot get this part of the data and recruit 
this diathesis into consideration. We have to say that this is really a pity.

\section{Conclusion}

Younger and older GC patients had significant differences in clinicopathological features, and younger GC patients had much better overall survival, lower MLR and higher PNI than older GC patients. The number of metastatic lymph nodes was the only risk parameter for a prognosis in younger GC patients, and AJCC TNM stage, radicality and tumor size were independent risk parameters for a prognosis in older GC patients. We should take more effective treatments for younger GC patients with lymph nodes metastasis and pay more attention to the nutritional problems of older GC patients.

\section{Disclosure}

The authors report no conflicts of interest in this work.

\section{References}

1. Torre LA, Bray F, Siegel RL, Ferlay J, Lortet-Tieulent J, Jemal A. Global cancer statistics, 2012. CA Cancer J Clin. 2015;65(2):87-108.

2. Saito H, Takaya S, Fukumoto Y, Osaki T, Tatebe S, Ikeguchi M. Clinicopathologic characteristics and prognosis of gastric cancer in young patients. Yonago Acta Med. 2012;55(3):57-61.

3. Park HJ, Ahn JY, Jung HY, et al. Clinical characteristics and outcomes for gastric cancer patients aged 18-30 years. Gastric Cancer. 2014;17(4): 649-660.

4. Isobe $\mathrm{Y}$, Nashimoto A, Akazawa K, et al. Gastric cancer treatment in Japan: 2008 annual report of the JGCA nationwide registry. Gastric Cancer. 2011;14(4):301-316.

5. Takatsu Y, Hiki N, Nunobe S, et al. Clinicopathological features of gastric cancer in young patients. Gastric Cancer. 2016;19(2):472-478.

6. Liu S, Feng F, Xu G, et al. Clinicopathological features and prognosis of gastric cancer in young patients. BMC Cancer. 2016;16:478.

7. Schlesinger-Raab A, Mihaljevic AL, Egert S, et al. Outcome of gastric cancer in the elderly: a population-based evaluation of the Munich Cancer Registry. Gastric Cancer. 2016;19(3):713-722.

8. Townsley C, Pond GR, Peloza B, et al. Analysis of treatment practices for elderly cancer patients in Ontario, Canada. J Clin Oncol. 2005;23(16): 3802-3810.

9. Deng Q, He B, Liu X, et al. Prognostic value of pre-operative inflammatory response biomarkers in gastric cancer patients and the construction of a predictive model. J Transl Med. 2015;13:66.
10. Sakurai K, Tamura T, Toyokawa T, et al. Low preoperative prognostic nutritional index predicts poor survival post-gastrectomy in elderly patients with gastric cancer. Ann Surg Oncol. 2016;23(11):3669-3676.

11. Wu Y, Zhou BP. Inflammation: a driving force speeds cancer metastasis. Cell Cycle. 2009;8(20):3267-3273.

12. Edge SB, Compton CC. The American Joint Committee on Cancer: the 7th edition of the AJCC cancer staging manual and the future of TNM. Ann Surg Oncol. 2010;17(6):1471-1474.

13. Tavares SM, Junior Wde L, Lopes E, Silva MR. Normal lymphocyte immunophenotype in an elderly population. Rev Bras Hematol Hemoter. 2014;36(3):180-183.

14. Lindblad M, Ye W, Rubio C, Lagergren J. Estrogen and risk of gastric cancer: a protective effect in a nationwide cohort study of patients with prostate cancer in Sweden. Cancer Epidemiol Biomarkers Prev. 2004;13(12):2203-2207.

15. Ohtani M, García A, Rogers AB, et al. Protective role of 17 betaestradiol against the development of Helicobacter pylori-induced gastric cancer in INS-GAS mice. Carcinogenesis. 2007;28(12):2597-2604.

16. Qin J, Liu M, Ding Q, et al. The direct effect of estrogen on cell viability and apoptosis in human gastric cancer cells. Mol Cell Biochem. 2014;395(1-2):99-107.

17. Kath R, Fiehler J, Schneider CP, Höffken K. Gastric cancer in very young adults: apropos four patients and a review of the literature. J Cancer Res Clin Oncol. 2000;126(4):233-237.

18. Wesołowska M, Pawlik P, Jagodziński PP. The clinicopathologic significance of estrogen receptors in human gastric carcinoma. Biomed Pharmacother. 2016;83:314-322.

19. Jaspers VK, Gillessen A, Quakernack K. Gastric cancer in pregnancy: do pregnancy, age or female sex alter the prognosis? Case reports and review. Eur J Obstet Gynecol Reprod Biol. 1999;87(1):13-22.

20. Saeidi H, Nasiri MR, Shahidsales S, et al. Evaluation of estrogen receptor expression and its relationship with clinicopathologic findings in gastric cancer. Adv Biomed Res. 2015;4:177.

21. Haruma K, Komoto K, Kamada T, et al. Helicobacter pylori infection is a major risk factor for gastric carcinoma in young patients. Scand $J$ Gastroenterol. 2000;35(3):255-259.

22. Hirahashi M, Yao T, Matsumoto T, et al. Intramucosal gastric adenocarcinoma of poorly differentiated type in the young is characterized by Helicobacter pylori infection and antral lymphoid hyperplasia. Mod Pathol. 2007;20(1):29-34.

23. Kong X, Wang JL, Chen HM, Fang JY. Comparison of the clinicopathological characteristics of young and elderly patients with gastric carcinoma: a meta analysis. J Surg Oncol. 2012;106(3):346-352.

24. Wada N, Kurokawa Y, Miyazaki Y, et al. The characteristics of the serum carcinoembryonic antigen and carbohydrate antigen 19-9 levels in gastric cancer case. Surg Today. 2017;47:227-232.

25. Liang Y, Wang W, Fang C, et al. Clinical significance and diagnostic value of serum CEA, CA19-9 and CA72-4 in patients with gastric cancer. Oncotarget. 2016;7(31):49565-49573.
OncoTargets and Therapy

\section{Publish your work in this journal}

OncoTargets and Therapy is an international, peer-reviewed, open access journal focusing on the pathological basis of all cancers, potential targets for therapy and treatment protocols employed to improve the management of cancer patients. The journal also focuses on the impact of management programs and new therapeutic agents and protocols on

\section{Dovepress}

patient perspectives such as quality of life, adherence and satisfaction. The manuscript management system is completely online and includes a very quick and fair peer-review system, which is all easy to use. Visit http://www.dovepress.com/testimonials.php to read real quotes from published authors. 